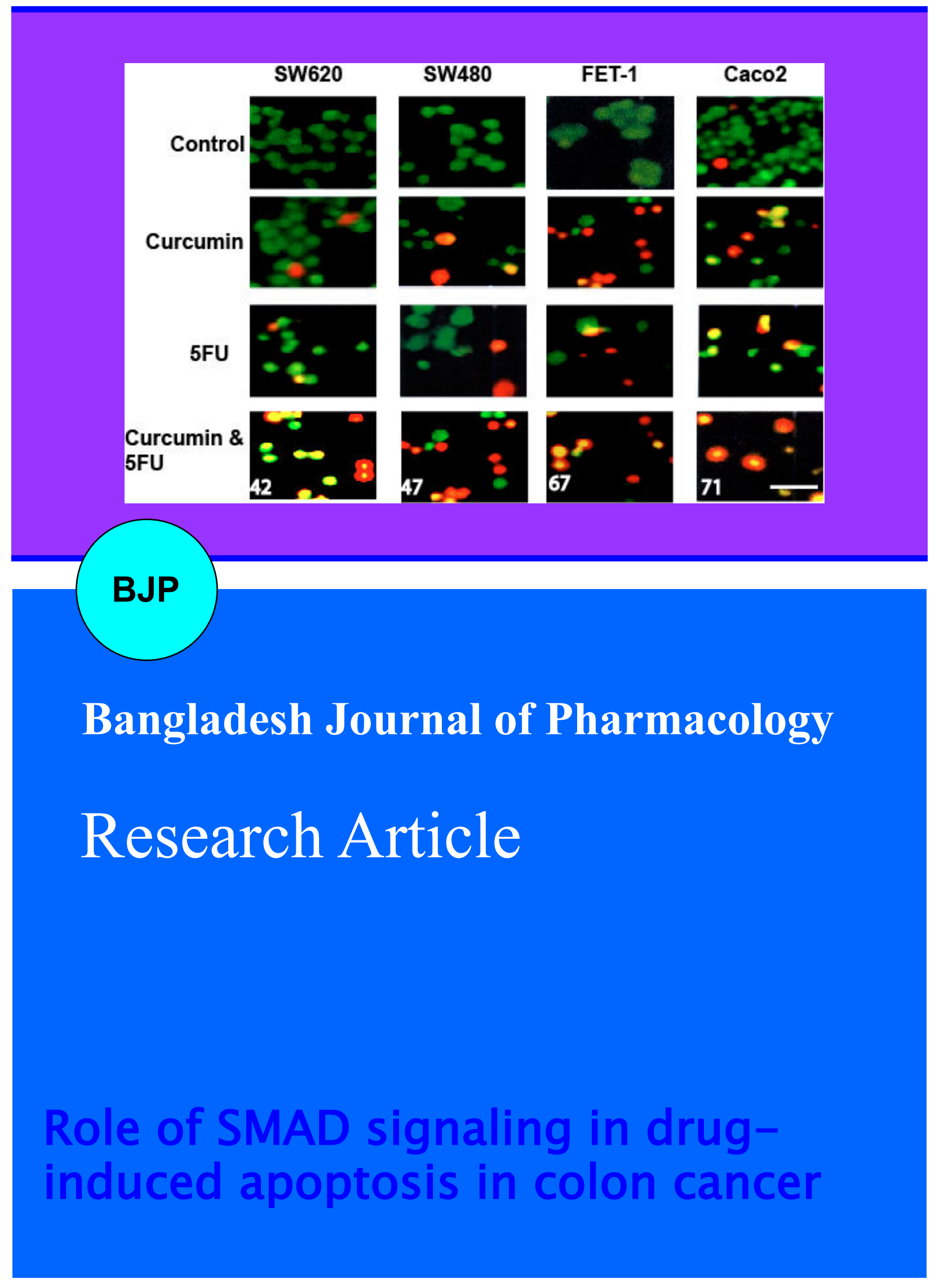




\title{
Role of SMAD signaling in drug-induced apoptosis in colon cancer
}

\author{
Bin Ren', Chong Liu², Li Hua Wu³, Peng Jin¹, Ping Li4, Yu Jie Liu' and Qing Bo Yu5 \\ ${ }^{1}$ Department of Anorectal Surgery, The Affiliated Hospital of WeiFang Medical University, Shandong 261031, China; \\ ${ }^{2}$ Department of Gastrointestinal Surgery, The Affiliated Hospital of WeiFang Medical University, Shandong 261031, \\ China; ${ }^{3}$ Department of Public Health, The Affiliated Hospital of WeiFang Medical University, Shandong 261031, \\ China; ${ }^{4}$ Department of Pathology, Weifang Nursing Vocational College, Shandong 261031, China; ${ }^{5}$ Department of \\ Thyroid Breast Surgery, The Affiliated Hospital of WeiFang Medical University, Shandong 261031, China.
}

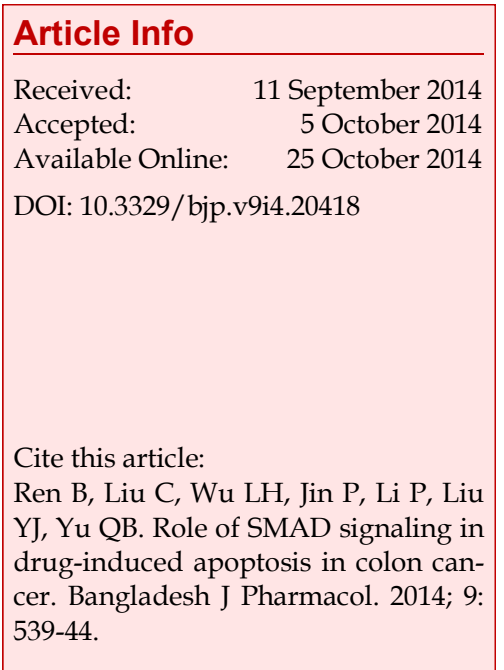

\begin{tabular}{|l|}
\hline Abstract \\
Aberrations in SMAD signaling have been widely associated with colon \\
cancer progression. However, their significance in modulating drug response \\
remains unknown. In this study we investigated the response of chemothera- \\
peutic drugs in colon cancer cells that are with (SW620 and SW480) and \\
without aberrations (FET-1 and Caco2) in SMAD signaling. These results \\
showed that curcumin, 5-fluorouracil and their combinations induced less cell \\
death in SMAD signaling non-responsive cells, while the apoptosis was ( $>2$ \\
fold) higher in SMAD responsive cells. Furthermore, these results showed \\
that the apoptosis in SMAD non-responsive cells was reversed and increased \\
$(\sim 2$-fold) upon ectopic expression of SMAD-4. Finally, correlation with \\
patients undergoing chemotherapy for colon cancer showed a strong \\
association between SMAD aberration and tumor relapse in chemotherapy. \\
Overall, the present data showed that SMAD signaling modulates the drug \\
responses and their signaling status should be considered while designing \\
chemotherapy regimen for colon cancer patients.
\end{tabular}

\section{Introduction}

SMAD family of proteins is implicated in TGF- $\beta$ signaling mediated epithelial growth inhibition (Massague et al., 2008). Upon ligand binding to TGF- $\beta$ receptors (TGFBRI and II), phosphorylation of receptor-activated SMADs (R-SMADs), SMAD2 and SMAD3, occur, which then bind to the common mediator SMAD (Co-SMAD) SMAD4 (Abdollah et al., 1997; Souchelnytskyio et al., 1997; Shi et al., 1997; Chacko et al., 2001 and 2004). The resulting complex then relocates into the nucleus and regulates the expression of many genes. This process could also be regulated by another inhibitory SMAD, SMAD7. Mutations causing aberrations in this signaling process are common in human malignancies including colorectal cancer (CRC). In sporadic CRCs, SMAD4 and TGFBR2 mutations are associated with $~ 10$ and $15 \%$ of patients respectively (Fleming et al., 2013).
The SMAD proteins are highly homologous with 2 conserved regions, an N-terminal Mad homology domain-1 (MH1) and a C-terminal Mad homology domain-2 (MH2) (Frederick et al., 2002; Miyazono et al., 2000). The MH1 domain regulates the nuclear import, DNA binding and transcriptional activity, while the MH2 domain mediates SMAD protein homoand hetero-oligomerization, and cytoplasmic anchoring (Frederick et al., 2002; Miyazono et al., 2000). Mutations in SMADs, in particular SMAD4, show a characteristic distribution with respect to its domain structure. The majority of changes cluster in the MH2 domain and often altering SMAD4 hetero-oligomerization with the R-SMADs (Fleming et al., 2013).

Despite the SMAD protein functions is known in the onset and the progression of CRC cancer, evidence for their role in modulating the drug responses is limited. 
Altered NF-kB signaling due to abnormal SMAD signaling has been recently implicated with CRC cancer cell survival (Grau et al., 2006). However, a direct evidence for SMAD signaling to modulate the druginduced apoptosis is not known.

In the present study, the SMAD signaling activation using SMAD4 protein levels as readout in drug induced apoptosis in colon cancer cells were studied.

\section{Materials and Methods}

\section{Cell culture, treatments and over expression}

Human colo-rectal cells (Caco2, FET, SW620 and SW480) were purchased from ATCC and maintained in DMEM (Invitrogen, NY, USA) supplemented with 10\% FBS. The drugs (curcumin and 5-fluorouracil) were purchased from Sigma, MO, USA. $5 \mu \mathrm{M}$ treatments were given to cells in all experiments, as it is the lowest of all $\mathrm{IC}_{50}$ concentration determined for all drug treatments in the tested cell lines (data not shown). For SMAD4 over-expression experiments, SMAD4-EGFPC1 plasmid was constructed and transfected using lipofectamine 2000 (Invitrogen, NY, USA), and enriched by Geniticin selection (Invitrogen, USA).

\section{Viability assay}

The growth-inhibitory effects of drugs were examined using a 3, 4, 5-dimethyl-2H-tetrazolium bromide assay (MTT; Sigma-Aldrich), as per manufacturer's instructions. Briefly, equal numbers of cells were incubated with drugs or DMSO treatments for 24 hours, followed by additional incubation with MTT for 4 hours. The MTT formazan was then measured and used as a quantitative measure of cell viability. The value obtained in control was set as reference and relative difference in percent viability is used to efficacy of drug treatments.

\section{Ethidium bromide and acridine orange (Et-Br/AO) staining}

Live and apoptotic cells were differentiated from others by acridine orange (AO) and ethidium bromide (Et-Br) staining as described previously (Sang et al., 2014). Stained cells were analyzed immediately by counting from at least three random in each experiment. Mean value from at least three independent experiments were provided.

\section{Western blotting}

Total protein from cells was extracted in lysis buffer (Pierce, Rockford, IL, USA) and quantified using the Bradford method. A total of $30 \mu \mathrm{g}$ of protein was separated using 4-12\% Bis-Tris gel and then transferred to a PVDF membrane (Millipore, Bedford, MA). The membrane was blocked with $5 \%$ dry milk and incubated overnight at $4^{\circ} \mathrm{C}$ with rabbit antibodies against SMAD4 or actin (1:1000, Cell Signaling, USA). After subsequent incubation with secondary antibodies, membrane was developed with ECL (Pierce, USA) and detected using Bio Imaging Systems (UVP, USA). The relative protein levels were calculated based on actin protein as a loading control.

\section{Chemotherapy correlation}

Patient information, were conducted as per the institute's ethical guidelines and under the supervision of WeiFang Medical University, Shandong, China.

As evaluating the correlation between SMAD mutation and chemotherapy response was interesting, a random heterogeneous patient population info spanning with mutations identified and unidentified and also spanning patients with both positive chemotherapy response and also with patient conditions with tumor relapsing within three years since first chemotherapy were analyzed. Three year time frame was selected to demarcate the tumor relapse and recovery phenotype, based on the empirical observation of tumor relapse in patients. However, there is still a possibility of recovered patients to have a relapsed tumor later than three years, but per the needs of the study grouped based on three year time frame as most tumor relapse would occur well before this time frame. Also, the tumor relapse was based on the primary site- here it is colon cancer. The sample group included both male and female patient ranging in the age group of $>40$ years were included. In order to avoid any sample bias during statistical analysis it is ensured that SMAD mutations patient populations alone were maintained less than $20 \%$ of total population info $(\mathrm{N}=157)$.

\section{Statistical analysis}

Statistical analyses were performed using GraphPad Prism (Version 6). Data were analyzed using one-way ANOVA analysis and statistical significance was set at minimal value of $p<0.05$. All values are listed as mean \pm standard deviation.

\section{Results}

MTT assay was performed for evaluating their cell viability. The viability of SW620 and SW480 cells in curcumin alone treated cells was 71.1 and $69.2 \%$, with 5FU alone was 67.2 and $71 \%$, but with the combination curcumin - 5-fluorouracil was reduced to 48.2 and $44 \%$ respectively (Figure 1). Similarly, viability of FET and Caco 2 cells was 41 and $43 \%$ cells in curcumin alone treated conditions, 47 and $49 \%$ cells in 5-fluorouracil treated condition, and 23 and $19 \%$ in synergistic treatment conditions. These results indicate that the SMAD signaling responsive cell lines is more sensitive to drugs than SMAD signaling non-responsive cell lines. In addition, the drug combinations could induce more cell death than the individual treatments in all the 


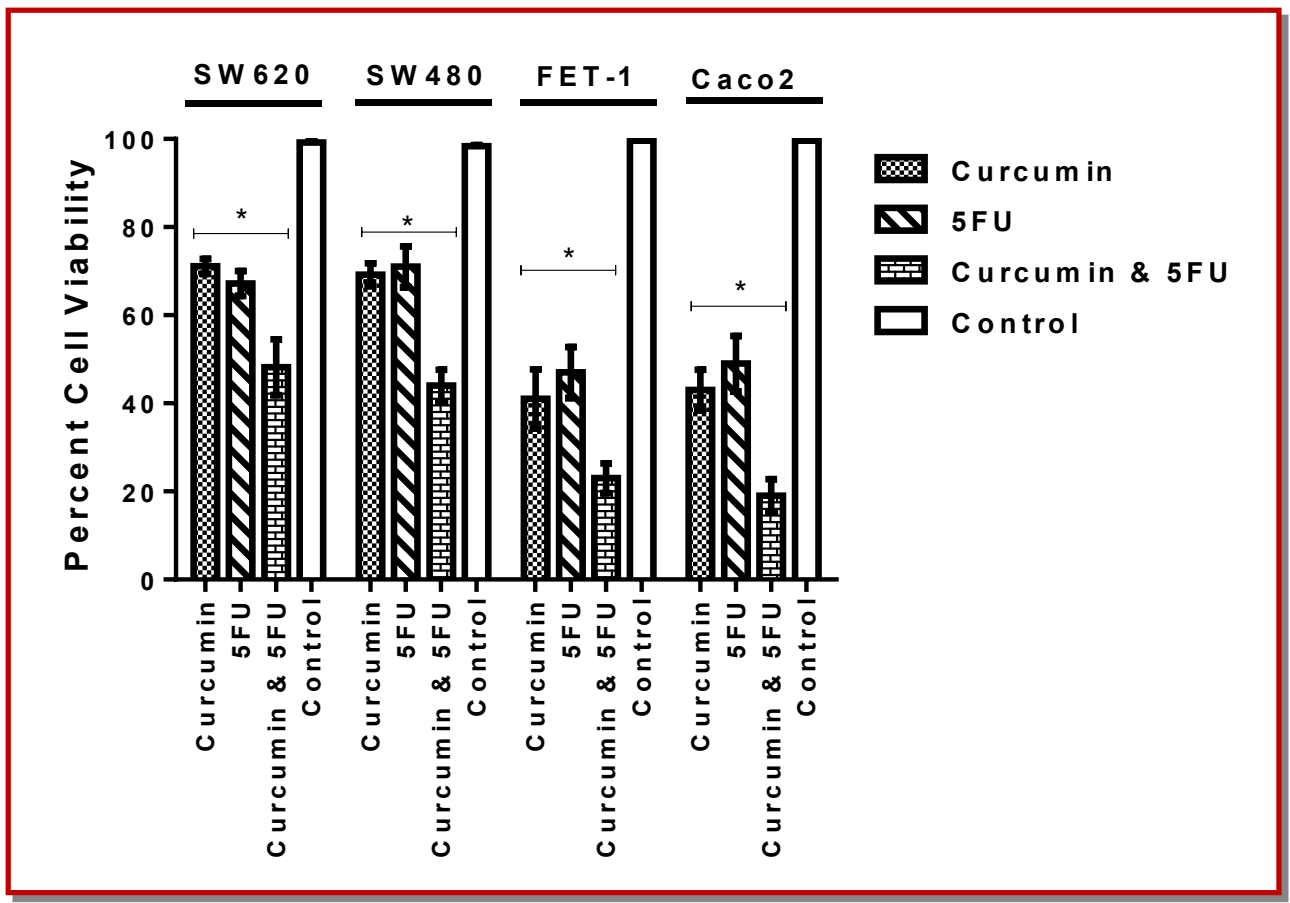

Figure 1: Evaluation of cell viability by MTT assay

MTT assay showing the effect of drug treatments on viability of cells. Cells were treated with individual drug treatments or combinations for 24 hours. Each point value represents a mean value from three independent experiments and represents a percent viability of cells remaining after drug treatments. SMAD responsive cells (FET-1 and Caco2) showed more response in general and further augmented in combination treatments. Statistical significance was seen as $\left({ }^{*} \mathrm{p}<0.01\right)$ between individual drug treatments and combinations

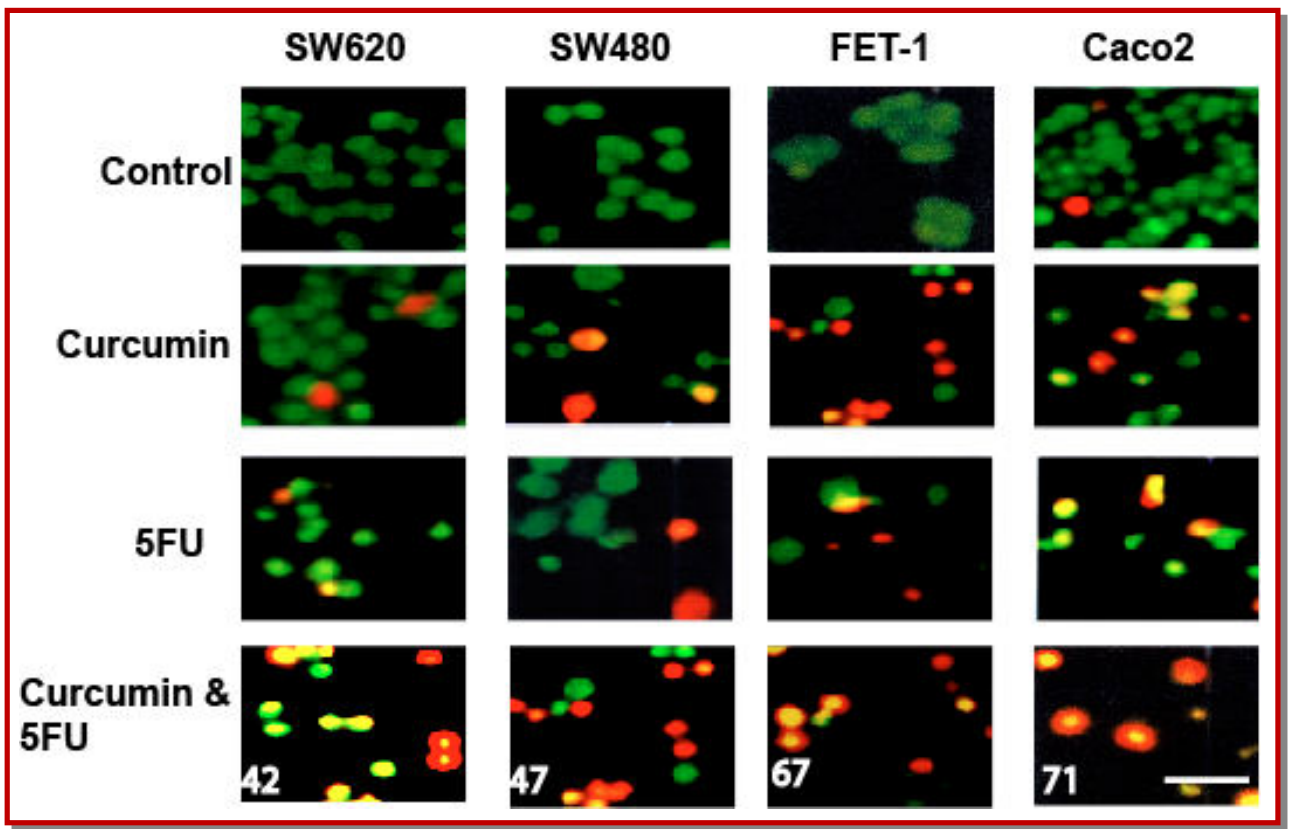

Figure 2: Morphological characteristics of cell death in drug induced apoptosis

Cells were treated with drugs and were evaluated for apoptosis using ET-Br/AO staining in all four cells (Green- Live cells; Orange/red- Dead cells). Increased apoptosis were observed in SMAD responsive cells (FET-1 and Caco2) both in individual and combination treatments than in SMAD non-responsive cells (SW620 and SW480). Inlet numerical value represents the mean percentage of orange/red cells (apoptotic cells) observe in synergistic drug treatments of 5FU and curcumin from three independent experiments. Scale bar $25 \mathrm{~mm}$. 


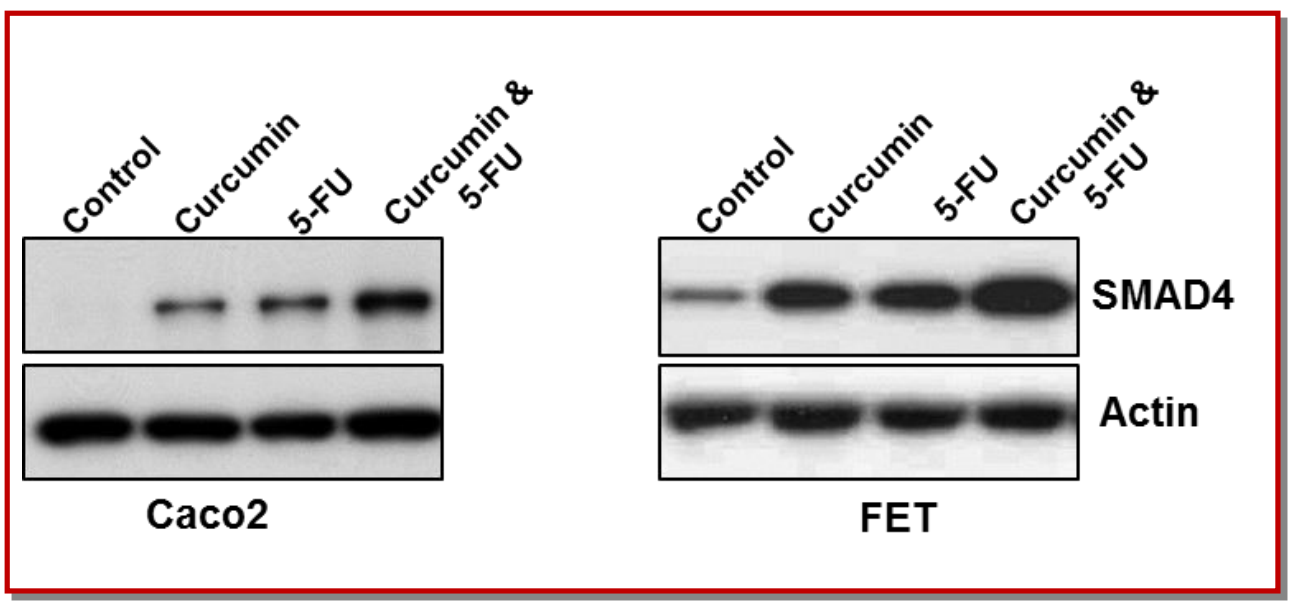

Figure 3: Evaluation of SMAD proteins in drug induced apoptosis

Cells were treated with drugs and were evaluated for SMAD 4 protein levels. SMAD4 levels were increased in FET-1 and Caco2 cells ( $>3$-fold). Beta-actin was used as a loading control

cell lines, but more prominently in SMAD responsive cell lines.

Ethidium bromide/acridine orange (Et-Br/AO) staining was done to investigate the quantitative and qualitative changes in nuclear condensation/apoptosis. The untreated cells showed green color, representing normal cells. At $5 \mu \mathrm{M}$ concentration of curcumin and 5- fluorouracil treatment on all cell lines, orange colored cells indicating DNA damage was observed. Cell shrinkage and nuclear condensation (smaller nuclei) were observed after treatments. However, there were only a few percentages of cells ( 20-30\%) showed these features in individual treatments in FET-1 and Caco2 cells and were minimal in SW620 and SW480 cells (< $20 \%$ ). In summary, individual treatments showed

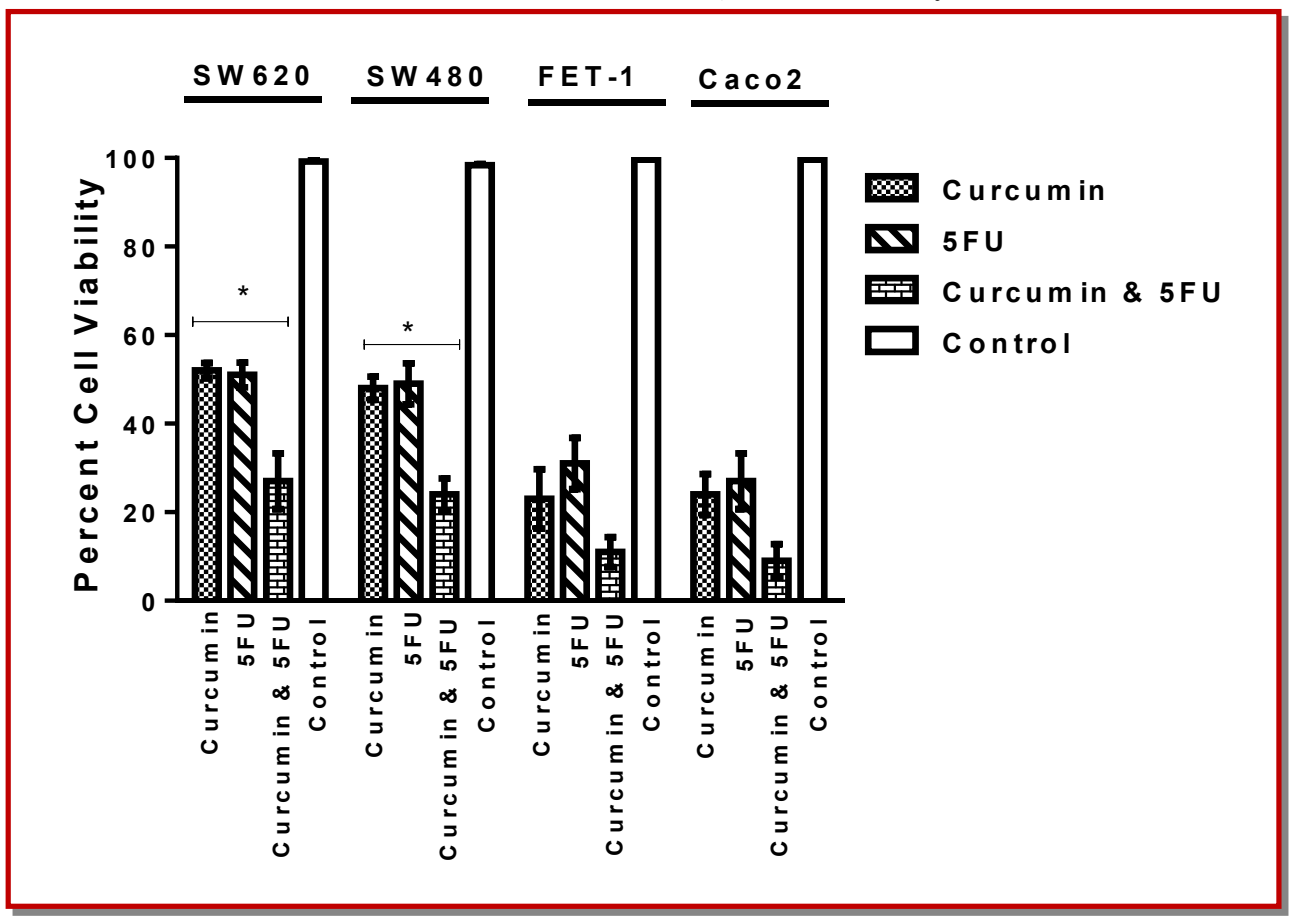

Figure 4: Overexpression of SMAD4 and improved drug response

SMAD4 overexpressing cells were treated with drugs and cell viability was evaluated by MTT assay. Each point value represents a mean value from three independent experiments and represents a percent viability of cells remaining after drug treatments. SMAD non-responsive cells SW620 and SW480 that are previously less prone to apoptosis now exhibited increased apoptosis. A similar trend of increased apoptosis were seen in other cells (FET-1 and Caco2) as well upon overexpressing SMAD4 in them. Statistical significance was seen as $\left({ }^{*} p<0.01\right)$ between individual drug treatments and combinations of only SW620 and SW480 cells alone as there were only few cell viability in any of the treatments in FET-1 and Caco2 cells 


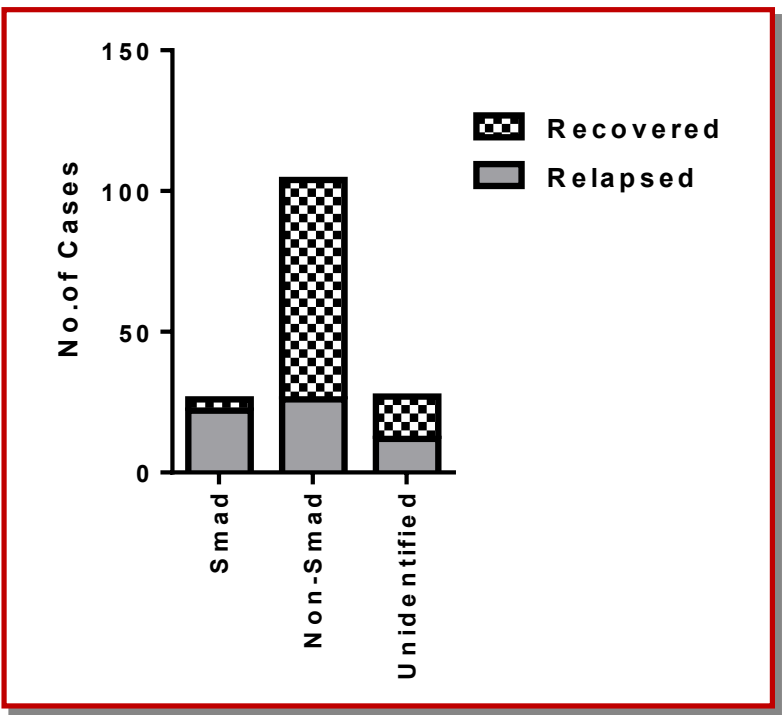

Figure 5: Association of chemotherapy response with causative mutations

Patients $(n=157)$ were grouped into three groups based on the mutations (SMAD family $(\mathrm{n}=26)$, non-SMAD $(\mathrm{n}=104)$ and unidentified $(n=27)$. The response seen in these patients after chemotherapy were graphed, which exhibit a strong associa-tion between SMAD mutations and tumor relapse

relatively lower percentage of cells positive for apoptosis (Figure 2). Furthermore, to some extent they also showed cell morphology without nuclear condensation or cell shrinkage suggesting for some necroticlike cell death. In contrast, synergistic treatments in all cells and more prominently included the apoptotic like changes, while there is no increase in necrotic like changes. This suggests that with more drug availability specific preferences to certain apoptosis causing pathways are prominent and hence there is an increase only in apoptosis.

In SW620 and SW480 cells SMAD 4 was undetectable due to the nature of the mutations. In contrast, Caco2 and FET-1 cells showed an up-regulation of $>3$-fold induction in individual treatments and further augmented in combination treatments (Figure 3). However, for other SMAD proteins such as SMAD 2/3 and SMAD7, a marginal up regulation and down-regulation was seen only in combination treatments respectively and there was no change in either SW620 or SW480 (data not shown). These data together suggest as SMAD4 as a critical modulator associated with drug induced effects in these cells.

Given that, SMAD4 expression is modulated upon drug treatments, we reasoned, whether SMAD4 could be mediating the drug-induced apoptosis in these cells. To evaluate, we ectopically overexpressed SMAD4 in these cells and subjected to drug treatments. Our results indicate that, ectopic expression of SMAD4 cells increased the response of cancer cells and increased the apoptosis in FET and Caco2 cells (Figure 4). More importantly, drug treatments induced increased apoptosis in SW620 and SW480 cells as well upon overexpressing SMAD4 in them. Taken together, these results suggest a direct role for SMAD4 in druginduced apoptosis.

Give with our observation in these cells, we also evaluated if there is any correlation between SMAD mutations and chemotherapy outcomes. We did correlative analysis with 157 patients (130-mutations previously identified) subjected to chemotherapy and their outcomes known. Our data showed a strong association (>95\%) between aberrant SMAD gene mutations (all SMAD genes) and tumor relapse after chemotherapy (Figure 5), suggesting that SMAD signaling is critical to mediate effective chemotherapy. Although, our cell line data indicate that only SMAD4 is largely changed with tested drug treatments (FET-1 and $\mathrm{Caco} 2$ cells), our patient-treatment information analysis showed that mutations in other SMAD genes were also associated with the tumor relapse phenotype. We speculate that other SMAD gene mutations could impact on SMAD4 functions and cause impaired response in chemotherapy.

\section{Discussion}

Colon cancer continues to remain as one of the major malignancy in most countries (Labianca et al., 2010). Abnormalities in SMAD signaling have a major role in the development of colon cancer (Massague et al., 2008; Fleming et al., 2013). In the present study the efficiency of drugs in inducing apoptosis was analyzed in four colon cancer cell lines, FET, Caco2, and two related SMAD nonresponsive cells, SW620 and SW480. The drugs (5-fluorouracil and curcumin) were selected based on their utility in CRC chemotherapy or their ability to induce apoptosis in a wide range of cancer cells. The MTT assay results show that both drugs were able to decrease the viability and the effects were more in the combination treatments. Earlier reports have shown that curcumin can induce apoptosis in many cancer cell lines (Hossain et al., 2012). However, in colon cancer cells it remains largely unknown. On the other hand, 5-fluorouracil has been widely used for colon cancer chemotherapy, and discrepancies in outcomes are observed (Pasetto et al., 2006). These data indicate that both the drugs mediated prominently only in a subset of cancer cells tested. SW620 and SW480 being SMAD non-responsive mutant lines showed less apoptosis induction, suggesting that SMAD proteins may mediate drug-induced apoptosis. In accordance with the induction of increased apoptosis by these drugs the expression levels of SMAD proteins is also positively modulated in SMAD responsive cells. The SMAD dependence is further confirmed as the phenotype is reversed and increased cell death is seen upon over expression of SMAD4 in SW620 and SW480. 
SMAD proteins are known to modulate to wide range of processes and are associated with many signaling pathways such as NF-kappa B, PI3K/AKT pathways (Grau et al., 2006; Zhang et al., 2013). Dissecting the pathways with the context of drug induced responses would be of more importance to understand the role SMAD in mediating the apoptosis. Most anticancer drugs induce DNA damage and apoptosis in by inducing single and double strand DNA breaks (ssb and $\mathrm{dsb}$, respectively. It is known that if a chromosomal break has been created, the tumor suppressor pathway genes (TSG) such as, p53 level rises, gets stabilized and induces cell cycle arrest and apoptosis (Lai et al., 2012). Although, further investigations are required on TSG levels and its relation with SMAD signaling, our data suggest that a classical DNA damage response mediated by TSG to cause apoptosis may depend on SMADs, at least in the colon cancer suggesting for further modulations of chemotherapy. This hypothesis is further augmented by strong association of tumor relapse and SMAD signaling conditions in patients.

Overall, the present data suggest that SMAD signaling mediate the drug induced apoptosis in colon cancer cells and also possibly mediate the chemotherapy response in patients of colon cancer, and hence could be used as predictive tool and design chemotherapy accordingly.

\section{Contribution}

First two authors contributed equally in the study.

\section{References}

Abdollah S, Macias-Silva M, Tsukazaki T, Hayashi H, Attisano L, Wran JL. TBRI phosphorylation of SMAD2 on Ser465 and Ser467 is required for SMAD2-SMAD4 complex formation and signaling. J Biol Chem. 1997; 272: 27678-85.

Chacko BM, Qin B, Correia JJ, Lam SS, de Caestecker MP, Lin $\mathrm{K}$. The L3 loop and C-terminal phosphorylation jointly define SMAD proteintrimerization. Nat Struct Biol. 2001; 8: 248-53.

Chacko BM, Qin BY, Tiwari A, Shi G, Lam S, Hayward LJ, De Caestecker M, Lin K. Structural basis of heteromeric SMAD protein assembly in TGF-b signaling. Mol Cell. 2004; 15: 813-
23.

Fleming NI, Jorissen RN, Mouradov D, Christie M, Sakthianandeswaren A, Palmieri M, Day F, Li S, Tsui C, Lipton L, Desai J, Jones IT, McLaughlin S, Ward RL, Hawkins NJ, Ruszkiewicz AR, Moore J, Zhu HJ, Mariadason JM, Burgess AW, Busam D, Zhao Q, Strausberg RL, Gibbs P, Sieber OM. SMAD2, SMAD3 and SMAD4 mutations in colorectal cancer. Cancer Res. 2013; 73: 725-35.

Frederick JP, Wang XF Smads "freeze" when they ski. Structure $2002 ; 10: 1607-11$.

Grau AM, Datta PK, Zi J, Halder SK, Beauchamp RD. Role of Smad proteins in the regulation of NF-kappaB by TGF-beta in colon cancer cells. Cell Signal. 2006; 18: 1041-50.

Hossain DM, Bhattacharyya S, Das T, Sa G. Curcumin: The multi-targeted therapy for cancer regression. Front Biosci (Schol Ed). 2012; 4: 335-55.

Labianca R, Beretta GD, Kildani B, Milesi L, Merlin F, Mosconi S, Pessi MA, Prochilo T, Quadri A, Gatta G, de Braud F, Wils J. Colon cancer. Crit Rev Oncol Hematol. 2010; 74: 106-33.

Lai D, Visser-Grieve S, Yang X. Tumour suppressor genes in chemotherapeutic drug response. Biosci Rep. 2012; 32: 36174 .

Massague J. TGFb in cancer. Cell 2008; 134: 215-30.

Miyazono K, ten Dijke P, Heldin CH. TGF-beta signaling by Smad proteins. Adv Immunol. 2000; 75: 115-57.

Pasetto LM, D'Andrea MR, Jirillo A, Rossi E, Monfardini S. Stable disease in advanced colorectal cancer: Therapeutic implications. Anti-cancer Res. 2006; 26: 511-22.

Sang HQ, Gu JF, Yuan JR, Zhang MH, Jia XB, Feng L. The protective effect of smilax glabra extract on advanced glycation end products-induced endothelial dysfunction in HUVECs via RAGE-ERK1/2-NF-kB pathway. J Ethnopharmacol. 2014; 155: 785-95.

Souchelnytskyi S, Tamaki K, Engstrom U, Wernstedt C, ten Dijke P, Heldin CH. Phosphorylation of Ser465 and Ser467 in the Cterminus of SMAD2 mediates interaction with SMAD4 and is required for transforming growth factor-beta signaling. J Biol Chem. 1997; 272: 28107-15.

Shi Y, Hata A, Lo RS, Massague J, Pavletich NP. A structural basis formutational inactivation of the tumour suppressor SMAD4. Nature 1997; 388: 87-93.

Zhang L, Zhou F, ten Dijke P. Signaling interplay between transforming growth factor- $\beta$ receptor and PI3K/AKT pathways in cancer. Trends Biochem Sci. 2013; 38: 612-20. 